\title{
Prevalencia de lesión del ligamento colateral medial de la rodilla valorada por resonancia magnética
}

\section{Prevalence of injury of the medial collateral ligament of the knee assessed by magnetic resonance}

\author{
Aguirre-Rodríguez VH, ${ }^{*}$ Valdés-Montor JF, ${ }^{\ddagger}$ Valero-González FS, ${ }^{\S}$ Santa-María-Gasca NE, \\ Gómez-Pérez MG, "Sánchez-Silva MC," Zúñiga-Isaac C, "Pérez-Mora HE," Mejía-Terrazas GE**
}

Hospital Ángeles Pedregal.

RESUMEN. Introducción: El ligamento colateral medial (LCM) es uno de los principales estabilizadores de la rodilla, pero su lesión se presenta en conjunto con otras lesiones ligamentarias. Objetivo: Determinar la prevalencia de lesiones del LCM por resonancia magnética, sus grados y lesiones asociadas en nuestra institución. Material y métodos: Estudio retrospectivo de Enero a Abril de 2018, se evaluaron resonancias magnéticas de rodilla donde se presentó lesión del LCM para evaluar grado y tipo de lesiones asociadas. Resultados: Se incluyeron 368 estudios, prevalencia de lesión aislada del LCM de 3.07\%, una grado I y una grado II, la prevalencia de lesiones de LCM concomitantes fue de $17.66 \%$, grado I (75\%), grado II (15\%) y grado III (3\%). Las lesiones asociadas fueron lesión del menisco medial (46.15\%), lesión del ligamento cruzado anterior (30.7\%), contusión ósea aislada (18.46\%), lesiones condrales (37.58\%), lesión de vasto medial (14.51\%), lesión del retináculo medial patelar (14.51\%), lesión del vasto lateral $(9.23 \%)$, lesión del ligamento cruzado posterior (6.15\%), lesión del menisco lateral (4.61\%), tenosinovitis banda iliotibial (4.61\%), fractura de avulsión de la faceta
ABSTRACT. Introduction: The medial collateral ligament (MCL) is one of the main stabilizers of the knee, but its injury occurs in conjunction with other ligaments. Objective: To determine the prevalence of MCL lesions by magnetic resonance imaging, their degrees and associated lesions in our institution. Material and Methods: Retrospective study from January to April 2018 where KNEE MRIs were evaluated where the MCL lesion was presented to evaluate the degree and type of associated injuries. Results: We included 368 studies, prevalence of isolated MCL lesion of $3.07 \%$, grade I and grade II, prevalence of concomitant MCL lesions was $17.66 \%$ grade I (75\%), grade II (15\%) and grade III (3\%). Associated injuries were medial meniscus injury (46.15\%), anterior cruciate ligament injury (30.7\%), isolated bone contusion (18.46\%), chodral injuries (37.58\%), medial vastus injury (14.51\%), patellar medial retinacular injury (14.51\%), vastus lateral injury (9.23\%), posterior cruciate ligament injury (6.15\%), lateral meniscus injury (4.61\%), iliotibial band tenosynovitis (4.61\%), medial facet avulsion fracture (3.07\%), Pes Anserine tenosynovitis (3.07\%). Conclusion:

\section{Nivel de evidencia: IV}

* Reconstrucción Articular, S.C. Clínica de Cadera y Rodilla, Hospital Ángeles del Pedregal; Profesor Adjunto, curso de Alta Especialidad en Reconstrucción Articular de Hombro y Codo, ULSA-HAP.

${ }^{\ddagger}$ Residente de Alta Especialidad en Reconstrucción Articular de Hombro y Codo, ULSA-HAP.

$\S$ Clínica de Hombro y Codo, Socio Médico Director, Hospital Ángeles del Pedregal; Profesor Titular, curso de Alta Especialidad en Reconstrucción Articular de Hombro y Codo, ULSA-HAP.

^ Director médico de resonancia magnética del Hospital Ángeles del Pedregal, Profesor titular de Alta Especialidad en Resonancia Magnética de Cuerpo Completo. UNAM-HAP.

" Residente de Alta Especialidad en Medicina de Resonancia Magnética de Cuerpo Completo. UNAM-HAP.

** Médico Anestesiólogo-Algólogo, Maestro en Ciencias Médicas, Clínica del Dolor Hospital Ángeles México.

Correspondencia:

Víctor Hugo Aguirre-Rodríguez

E-mail: cadera.rodilla.hap@gmail.com

Recibido: 15-06-2020. Aceptado: 20-08-2021.

Citar como: Aguirre-Rodríguez VH, Valdés-Montor JF, Valero-González FS, Santa-María-Gasca NE, Gómez-Pérez MG, Sánchez-Silva MC, et al. Prevalencia de lesión del ligamento colateral medial de la rodilla valorada por resonancia magnética. Acta Ortop Mex. 2021; 35(3): 271-275. https://dx.doi.org/10.35366/102366

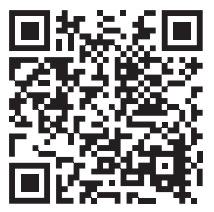


medial (3.07\%), tenosinovitis de la Pes Anserinus (3.07\%). Conclusión: Prevalencia de $17.66 \%$ de lesiones del LCM en nuestro hospital por resonancia magnética, predominan los dos primeros grados con un espectro amplio de lesiones asociadas de la rodilla.

Palabras clave: Ligamento colateral medial, esquina posteromedial, prevalencia, lesión.

\section{Introducción}

El ligamento colateral medial es uno de los principales estabilizadores mediales de la rodilla, su estructura está constituida por tres haces, el ligamento colateral medial superficial, ligamento colateral medial profundo y el ligamento posterior oblicuo. ${ }^{1,2,3}$ Secundariamente este complejo ligamentario también proporciona estabilidad rotatoria y

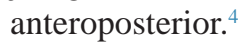

El ligamento posterior oblicuo refuerza la cápsula posterior y es una extensión fibrosa de la porción distal del semimembranoso, entre $0^{\circ}$ y $30^{\circ}$ de flexión de la rodilla es un estabilizador medial secundario y actúa también como un rotador interno. A medida que la flexión de la rodilla aumenta, al realizar rotación interna la carga es transferida al LCM superficial recibiendo este último la mayor carga en rotación interna a $90^{\circ}$ de flexión.

El ligamento colateral medial profundo es un estabilizador secundario de las fuerzas en valgo; a $60^{\circ}$ de flexión la porción menisco tibial es la que proporciona mayor estabilidad, en el resto de los grados de flexión su mayor estabilidad será dada por la porción menisco femoral. ${ }^{1,5}$

La incidencia de las lesiones del ligamento colateral medial van desde 0.24 en población general hasta 18.65 por cada 1,000 habitantes en grupos de personas que se encuentran en un ámbito deportivo, siendo de 7.27 en la serie de Roach y colaboradores para las lesiones aisladas del LCM y de 5.36 para la población femenina. . $^{1,46}$

Las lesiones del LCM según la Asociación Médica Americana (AMC por sus siglas en inglés), se dividen en tres grados: el grado I se presenta con hipersensibilidad, pero sin hiperlaxitud. El grado II se manifiesta con sensibilidad localizada y desgarro parcial del LCM y de las fibras oblicuas del ligamento posterior, las cuales continúan opuestas, puede existir laxitud no patológica. En el grado III las fibras tienen una disrupción completa y existe hiperlaxitud al aplicar una fuerza en valgo. Esta clasificación puede ser subjetiva, ya que depende de la capacidad de relajación del paciente y la capacidad del médico para aplicar la fuerza en valgo. ${ }^{1,7}$ También se pueden clasificar radiológicamente cuando se compara la apertura medial de la articulación contra la extremidad contralateral. El grado I presenta una diferencia de 3 a 5 $\mathrm{mm}$, el grado II va de 6 a $10 \mathrm{~mm}$ y el grado III con una diferencia $>10 \mathrm{~mm}$.
Prevalence of $17.66 \%$ of the MCL injuries in our hospital by magnetic resonance, the first 2 degrees predominate, with a wide spectrum of associated knee injuries.

Keywords: Medial collateral ligament, posteromedial corner, prevalence, injury.

En nuestro grupo de trabajo consideramos que las lesiones del LCM de rodilla son muy frecuentes y su diagnóstico imagenológico podría pasar desapercibido, las lesiones aisladas que requieren tratamiento quirúrgico son raras, las lesiones del LCM por lo regular están relacionadas con otras lesiones ligamentarias, lesiones osteocondrales y/o meniscales y éstas normalmente son tratadas de forma quirúrgica.

Nuestro objetivo es determinar la prevalencia de una lesión del LCM de la rodilla en la población que acudió al servicio de resonancia magnética del hospital; conocer los grados e identificar las lesiones asociadas.

\section{Material y métodos}

Estudio retrospectivo, transversal, descriptivo y observacional de acuerdo a Méndez y colaboradores 2001. ${ }^{8}$ Para el procesamiento de la información se elaboró una base de datos en Excel de Microsoft y el análisis estadístico se realizó con el paquete computacional Statistical Package for the Social Sciences (SPSS) versión $24 .^{9}$

Se identificaron de forma retrospectiva todos los estudios de resonancia magnética de rodilla en el período de Enero a Abril de 2018 en el Servicio de Resonancia Magnética del hospital. Se realizaron los estudios en un resonador Phillips 3 Tesla o en el resonador General Electric de 1.5 Tesla de forma independiente.

Se incluyeron todos los casos de rodilla, de ambos géneros y se separaron los estudios que tuvieran lesiones LCM de cualquier grado, aisladas o combinadas con otras patologías. Se excluyeron todas las rodillas que presentaban inmadurez esquelética. Los protocolos en ambos resonadores constan de secuencias axiales, coronales y sagitales potenciadas en T1, T2, saturación grasa, secuencias especiales de patela y secuencias especiales para ligamento cruzado anterior.

EL método de valoración en resonancia magnética que se utilizó fue de acuerdo a la clasificación internacional de lesiones de ligamento medial ${ }^{10}$ (Tabla 1).

Las interpretaciones en imagenología fueron realizadas exclusivamente por la profesora titular del curso de alta especialidad en medicina de resonancia magnética de cuerpo completo. Está documentado en la literatura que la resonancia magnética en lesión del ligamento colateral medial muestra una sensibilidad de $94.4 \%$, especificidad de $100 \%$ y precisión de 95\%, ${ }^{11}$ dependiendo de quien la revise. 
Tabla 1: Clasificación internacional de lesiones de ligamento colateral medial de la rodilla.

\begin{tabular}{lll} 
Grado & Lesión & IRM \\
\hline I & $\begin{array}{l}\text { Ruptura microscóscopica del lig, sin inestabilidad. Tensión y dirección } \\
\text { conservadas con líquido periligamentario } \\
\text { Ruptura parcial de las fibras menor de } 80 \% \text {. Existe edema asociado a partes } \\
\text { blandas, periligamentarios e inestabilidad }\end{array}$ & Hipointenso en T1 e hipertenso en T2 y sat grasa \\
II & Ruptura de las fibras mayor de $80 \%$ & Intensidad de señal de las fibras suele estar retardas \\
III &
\end{tabular}
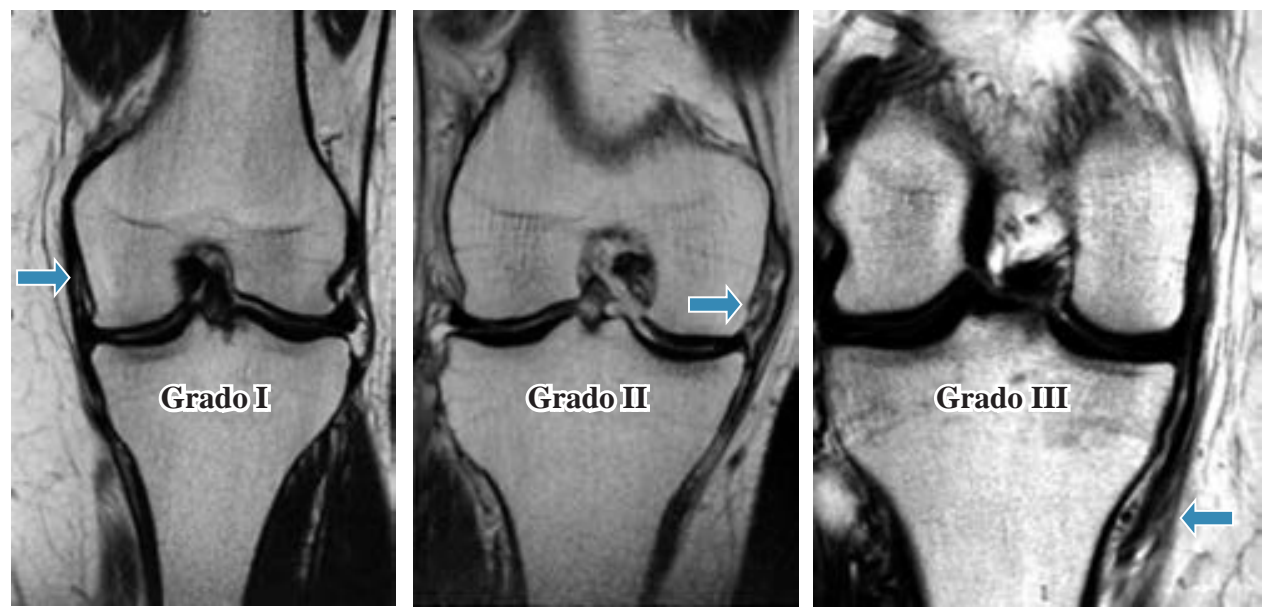

Figura 1:

Composición de los diferentes grados de lesión del ligamento colateral medial por resonancia magnética.

\section{Resultados}

Se examinaron 368 estudios de resonancia magnética de rodilla realizados en nuestro hospital, de los cuales sólo 65 (17.66\%) presentaron una lesión del ligamento colateral medial.

Tuvimos 37 casos (56.9\%) de género masculino y 28 casos (43.1\%) femeninos, la edad promedio de los pacientes fue de 45.64 años (con un rango de 19-80 años), existía un predominio de lateralidad mayor del lado izquierdo con 38 casos (58.46\%). Las lesiones encontradas de grado I fueron en 49 casos (75.38\%), de grado II, 10 casos $(15.38 \%)$ y de grado III, se detectaron dos casos $(3.07 \%)$, se presentaron cuatro casos $(6.15 \%)$ de tendinosis del LCM (Figura 1). Las lesiones asociadas halladas fueron lesiones del menisco medial en 30 casos (46.15\%), de las lesiones condrales juntas se hallaron 24 casos (36.91\%), de fémur fueron 11 casos (16.92\%), lesión condal de tibia en nueve casos (13.84\%) y lesiones condales de la rótula en cuatro casos (6.15\%), lesión del LCA en 20 casos (30.76\%), la contusión ósea se observó en 12 casos (18.46\%), de la lesión de vasto medial y lesión del retináculo medial se encontraron en nueve casos cada una (13.84\%), la lesión del vasto lateral en seis casos (9.23\%) y la lesión del LCP en cuatro casos cada una (6.15\%), la lesión del menisco lateral y la tenosinovitis de la banda iliotibial se observaron en tres casos (4.61\%), fractura de avulsión de la faceta medial y la tenosinovitis de la Pes Anserinus en dos casos (3.07\%) y las lesiones del vasto medial, del cuádriceps, lesión del tendón poplíteo, tendón patelar, ruptura del semimembranoso, de la cápsula articular así como las fracturas de la cabeza del peroné y del polo inferior de la patela sólo se encontraron en un caso $(1.53 \%)$ cada uno. Sólo se detectaron dos casos (3.07\%) que presentaban exclusivamente una lesión aislada del ligamento colateral medial y fueron uno de grado I y uno de grado II respectivamente.

\section{Discusión}

La literatura refiere que el mayor número de lesiones del LCM se presenta en pacientes jóvenes, de sexo masculino que practican deportes de contacto, el deporte asociado a un mayor número de lesiones es el fútbol americano, seguido de otros deportes como lucha, hockey, fútbol soccer, rugby, esquí, basquetbol, etc. En mujeres se asocia con más frecuencia a deportes como el rugby. ${ }^{4}$ Son lesiones asociadas principalmente a deportes de contacto ocasionadas por una contusión directa o maniobras con el pie fijo en el piso que provocan una deformidad en valgo de la rodilla, cuando se suma una fuerza rotacional se asocia a lesiones del ligamento oblicuo posterior.

La incidencia reportada de las lesiones del ligamento colateral medial va desde 0.24 en población general hasta 18.65 por cada 1,000 habitantes en grupos de personas que se encuentran en un ámbito deportivo, siendo de 7.27 en la serie de Roach y colaboradores para las lesiones aisladas del LCM y de 5.36 para la población femenina. .,4,6 $^{-1}$ 
La mayor parte de las lesiones son de bajo grado, según Roach y colaboradores en su serie éstas representaron 73\% del total de las lesiones aisladas del LCM, 23\% correspondieron a lesiones de grado II y sólo 4\% se asoció a lesiones de grado III. ${ }^{4}$

En nuestro estudio pudimos observar que tenemos una prevalencia de las lesiones del ligamento colateral medial por imagen en $17.66 \%$. Una debilidad del estudio es que no fue posible determinar los antecedentes deportivos, su nivel de actividad física y mecanismo de lesión. Sabiendo que es una de las lesiones que más frecuentemente se asocia a deportes de contacto, en nuestra serie de casos se observó que $75 \%$ de las lesiones del LCM eran grado I, seguidas de 15\% de grado II y $3 \%$ de grado III, muy parecida a la obtenida por Roach y colaboradores.

Para la valoración imagenológica del ligamento colateral medial, la mejor adquisición es la proyección coronal, la cual nos permite valorar la morfología, intensidad de señal, tensión, dirección y la inserción tibial y femoral. El ligamento colateral medial o ligamento colateral interno se observa hipointenso en todas las secuencias.

Las lesiones asociadas a desgarros del LCM deben ser siempre valoradas para definir pronóstico, tratamiento y tiempo de reintegración a la actividad deportiva. ${ }^{1}$ Aproximadamente $78.1 \%$ de las lesiones de grado III se asocian a lesiones de otras estructuras, de éstas 95\% corresponden a lesiones de LCA, se han encontrado asociadas hasta en $88 \%$ de los casos, siendo de vital importancia valorar la estabilidad del LCA previo a establecer un tratamiento definitivo. ${ }^{1,4}$ Otras estructuras como meniscos, cartílago y estructuras óseas pueden verse afectadas en combinación con las lesiones de LCM. ${ }^{3}$ Las lesiones meniscales susceptibles de reparación pueden ser tratadas mediante técnicas de reparación, de lo contrario se puede realizar meniscectomía. ${ }^{6}$

Clínicamente podemos encontrar edema, hipersensibilidad y equimosis en la región medial de la rodilla, cuando se asocia a hemartrosis es importante pensar en lesiones multiligamentarias. ${ }^{3}$ Además de la evaluación de la estabilidad en valgo, existen dos maniobras para valorar la integridad del LCM, la prueba de cajón anteromedial que se realiza flexionando la rodilla a $90^{\circ}$ mientras se rota el pie externamente entre $10^{\circ}$ y $15^{\circ}$ y se aplica una fuerza anteromedial a la rodilla. También podemos apreciar aumento en la rotación externa con la rodilla a $30^{\circ}$ y $90^{\circ}$ de flexión. ${ }^{1}$

Diferentes estudios han detectado una abundante vascularidad en el LCM que le confiere una alta tasa de cicatrización en el modelo clásico que comprende hemorragia, inflamación, reparación y remodelamiento. Existe un consenso que recomienda tratamiento conservador para las lesiones grado I o II con buenos resultados reportados en diferentes series en las que se complementa con órtesis, que limitan la flexión y la rotación externa, acompañado de distintos programas de rehabilitación física. ${ }^{1}$ El tiempo de reincorporación a la actividad física varía según el grado de lesión, oscilando en diferentes series entre 10.6 días y 2.4 meses para tratamiento conservador. ${ }^{1,2,4,10}$
Para las lesiones de grado III agudas, el tratamiento conservador puede ofrecer buenos resultados, en caso de inestabilidad severa o lesiones crónicas que coexisten con sintomatología se recomienda tratamiento quirúrgico así como para pacientes con lesiones combinadas. ${ }^{1}$ En los casos de lesiones de LCA asociados se ha observado que los resultados no ofrecen ninguna diferencia estadísticamente significativa entre el tratamiento quirúrgico de ambas lesiones y la reparación sólo del LCA. ${ }^{\text {}}$

Este estudio identificó en las lesiones del menisco medial una prevalencia de $46.15 \%$ de nuestros casos, seguida de las lesiones del LCA en 30.76\% y posteriormente la contusión ósea en $18.46 \%$, pero si sumamos las lesiones condrales de fémur, tibia y patela éstas pueden alcanzan hasta $36.91 \%$ en los casos encontrados, siendo más frecuentes que las lesiones del LCA. No contamos con estudios prospectivos más grandes y nuestra serie de casos estuvo limitada a un corto tiempo, lo que nos hace pensar que en un gran número de patologías en los esguinces de rodilla no se hace resonancia magnética.

Existe una nueva clasificación para las lesiones del ligamento colateral media basada en resonancia magnética y hallazgos clínicos, una guía para la selección de pacientes y de intervención temprana ${ }^{12}$ que empezaremos a utilizar en nuestro grupo.

\section{Conclusión}

Es nuestro primer reporte sobre las lesiones del ligamento colateral medial basado en la resonancia magnética de un hospital privado; su detección por imagen fue de $17.66 \%$, principalmente los dos primeros grados, con un espectro amplio de lesiones asociadas como la meniscopatía medial, la lesión del ligamento cruzado anterior y por supuesto, las lesiones condrales.

Referencias

1. Wijdicks CA, Griffith CJ, Johansen S, Engebretsen L, LaPrade RF. Injuries to the medial collateral ligament and associated medial structures of the knee. J Bone Jt Surg Ser A. 2010; 92(5): 1266-80. doi: 10.2106/JBJS.I.01229.

2. Smyth MP, Koh JL. Review article a review of surgical and nonsurgical outcomes of medial knee injuries. Sports Med Arthrosc Rev. 2015; 23(2): e15-22.

3. Menzer H, Treme G, Wascher D. Surgical treatment of medial instability of the knee. Sports Med Arthrosc Rev. 2015; 23(2): 77-84.

4. Roach CJ, Haley CA, Cameron KL, Pallis M, Svoboda SJ, Owens BD. The epidemiology of medial collateral ligament sprains in young athletes. Am J Sports Med. 2014; 42(5): 1103-9. doi: $10.1177 / 0363546514524524$.

5. Prince MR, Blackman AJ, King AH, Stuart MJ, Levy BA. Open anatomic reconstruction of the medial collateral ligament and posteromedial corner. Arthrosc Tech. 2015; 4(6): e885-90. doi: 10.1016/j.eats.2015.08.013.

6. Barrett IJ, Krych AJ, Pareek A, et al. Short- to mid-term outcomes of anatomic MCL reconstruction with Achilles tendon allograft after multiligament knee injury. Knee Surg Sports Traumatol Arthrosc. 2018; 26(10): 2952-59. doi: 10.1007/s00167-018-4843-4.

7. Varelas AN, Erickson BJ, Cvetanovich GL, Jr BRB. Medial collateral ligament reconstruction in patients with medial knee 
instability a systematic review. Orthop J Sports Med. 2017; 5(5): 2325967117703920. doi: 10.1177/2325967117703920.

8. Méndez RI, Namihira GD, Moreno AL, Sosa de MYC. El protocolo de investigación. Lineamiento para su elaboración y análisis. 2a edición. Editorial Trillas. México. 2001.

9. Van Belle G, Fisher DL, Heagerty JP, Lumley T. Biostatistics: a methodology for the health sciences. New York. WilleyInterscience. 2004.
10. Kramer DE, Miller PE, Berrahou IK, Yen Y-M, Heyworth BE. Collateral ligament knee injuries in pediatric and adolescent athletes. $J$ Pediatr Orthop. 2020; 40(2): 71-77. doi: 10.1097/BPO.0000000000001112.

11. Freitas A, Chew FS. Collateral ligament injury MRI. Drugs \& Diseases Radiology. 2015; 11.

12. Makhmalbaf H, Shahpari O. Medial colleteral ligament injury: a new classification bases on MRI and clinical findings. A guide for patient selection and early surgical intervention. Arch Bone Jt Surg. 2018; 6(1): 3-7. 